\title{
Isomerization of Hydrofluorocyclopentenes Promoted by Fluoride Anion
}

\author{
Chengping Zhang ${ }^{1}$, Ni Zhang ${ }^{1}$ Xiaoqing Jia ${ }^{1}$, Nan Li ${ }^{1}$, Hengdao Quan ${ }^{2 *}$ \\ ${ }^{1}$ Beijing Institute of Technology, Beijing, China \\ ${ }^{2}$ National Institute of Advanced Industrial Science and Technology (AIST), Tsukuba, Japan \\ Email: *hengdao-quan@aist.go.jp
}

How to cite this paper: Zhang, C.P., Zhang, N., Jia, X.Q., Li, N. and Quan, H.D. (2018) Isomerization of Hydrofluorocyclopentenes Promoted by Fluoride Anion. Green and Sustainable Chemistry, 8, 115-129. https://doi.org/10.4236/gsc.2018.81008

Received: November 21, 2017

Accepted: February 23, 2018

Published: February 26, 2018

Copyright (๑) 2018 by authors and Scientific Research Publishing Inc. This work is licensed under the Creative Commons Attribution International License (CC BY 4.0).

http://creativecommons.org/licenses/by/4.0/

(c) (i) Open Access

\begin{abstract}
The isomerization of hydrofluorocyclopentenes promoted by fluoride anion was investigated. It was found that two processes were responsible for interconversion of the isomers: an allylic syn-addition/elimination of fluoride anion that does not change the mutual positions of hydrogen atoms but is responsible for transfers of fluorine atoms, and a fluoride anion-assisted deprotonation/protonation which does not change the mutual positions of fluorine atoms but is responsible for transfers of hydrogen atoms. In the deprotonation, HF can easily capture excess fluoride anion to form $\mathrm{HF}_{2}^{-}$anion which can probably inhibit the protonation.
\end{abstract}

\section{Keywords}

Hydrofluorocyclopentene, Allylic Syn-Addition/Elimination,

Deprotonation/Protonation

\section{Introduction}

In order to fulfill the Montreal Protocol and the Kyoto Protocol, which mandate to phase out the applications of both ozone depleting substances (ODS) and high greenhouse substances in the field of chlorofluorocarbons (CFCs), many countries have looked for alternatives of CFCs. Hydrofluoroolefins (HFOs) have short atmospheric lifetimes, leading to distinct environmental benefits. Thus, HFOs have been considered as alternatives to HCFCs and HFCs. Heptafluorocyclopentenes, including 1H-heptafluorocyclopentene (F7E-1H), 3H-heptafluorocyclopentene $(\mathrm{F} 7 \mathrm{E}-3 \mathrm{H})$ and $4 \mathrm{H}$-heptafluorocyclopentene $(\mathrm{F} 7 \mathrm{E}-4 \mathrm{H})$, are one kind of alternatives that have zero ODP and low GWP. It is used as dry etching gas, fluorine-containing medicine intermediate, and hydrofluorocarbon-based solvent [1]. 
Today, HFOs can be synthesized by fluorine-chlorine exchange of hydrochlorofluoroolefins (HCFOs) [2], dehydrohalogenation of HFCs or HCFCs [3], hydrogenation of HCFOs or HFOs [4], addition of hydrofluoroalkyne with HF [5], or isomerization of HFOs [6]-[11]. In the above methods, the isomerization of HFOs plays an important key role in the synthesis of HFOs. 1,3,3,3-tetrafluoropropene (HFO-1234ze) has some momentous isomers, such as Z-1,3,3,3-tetrafluoropropene (Z-HFO-1234ze), E-HFO-1234ze, 1,1,3,3-tetrafluoropropene (HFO$1234 \mathrm{zc}$ ) and 2,3,3,3-tetrafluoropropene (HFO-1234yf), and they can be realized into the mutual transformation in the presence of various catalysts. Z-HFO1234ze was changed into E-HFO-1234ze catalyzed by fluorinated $\mathrm{Cr}_{2} \mathrm{O}_{3}, \mathrm{FeF}_{3}$, $\mathrm{AlF}_{3}$ or $0.5 \% \mathrm{Co} / \mathrm{C}$ at $20^{\circ} \mathrm{C}-100^{\circ} \mathrm{C}$ [7]. And E-HFO-1234ze was turned into Z-HFO-1234ze and a small amount of HFO- $1234 \mathrm{zc}$ at $200^{\circ} \mathrm{C}-550^{\circ} \mathrm{C}$ [8]. HFO$1234 \mathrm{zc}$ was isomerized into E-HFO-1234ze and Z-HFO-1234ze when promoted by $\mathrm{Cr}_{2} \mathrm{O}_{3}$ in the presence of $\mathrm{HF}$ at $250^{\circ} \mathrm{C}-280^{\circ} \mathrm{C}$ [11]. HFO- $1234 \mathrm{yf}$ was converted to E-HFO-1234ze and Z-HFO-1234ze promoted by $\mathrm{Cr}_{2} \mathrm{O}_{3}$ catalyst at $350^{\circ} \mathrm{C}$ [10]. In addition, Z-1,1,3,3,3-pentafluoropropene (Z-HFO-1225ye) was generated in the isomerization of E-HFO-1225ye promoted by $\mathrm{AlF}_{3}$ catalyst at $30^{\circ} \mathrm{C}$ [9]. And Z-1,1,1,4,4,4-hexafluoro-2-butene (Z-HFO-1336mzz) was produced by the isomerization of E-HFO-1336mzZ in the presence of fluorinated chromuium-based catalyst at $250^{\circ} \mathrm{C}[6]$.

The above isomerization technologies focused mainly on the isomerization between geometric isomers of HFOs [6] [7] [8] [9], while the isomerization between positional isomers of HFOs was rarely reported. The latter type of isomerization of HFOs always occurred at high temperature in the presence of special catalysts [10] [11], such as $\mathrm{Cr}_{2} \mathrm{O}_{3}$, which are always considered as business secrets, and for competitive reasons, details of these catalysts are probably not public. Until now, few literatures reported that the isomerization between positional isomers of HFOs promoted by simple catalysts occurred under mild conditions.

Here, we reported the isomerization of hydrofluorocyclopentenes (c5-HFOs) promoted by fluoride anion under mild conditions. The structures of c5-HFOs were confirmed by GC-MS, ${ }^{1} \mathrm{H}$ NMR and ${ }^{19} \mathrm{~F}$ NMR. Based on the results of our experiments, the mechanisms of the isomerizations of c5-HFOs in liquid-phase were proposed.

\section{Experimental}

\subsection{Chemicals}

$\mathrm{CCl}_{3} \mathrm{~F}$ (CFC-11) 99.0+\% was purchased from Synquest Labs, Lnc. Chloroform-d $\left(\mathrm{CDCl}_{3}\right)$ at 99.8 atom \%D, $\mathrm{H}_{2} 99.9 \%$ were obtained from Kanto Denka Co. (Japan). $\left(\mathrm{NH}_{4}\right)_{2} \mathrm{CO}_{3} 99.9 \%$, tetra-n-butylammonium bromide $99.8+\%$, $\mathrm{LiF} 99.8+\%$, $\mathrm{NaF}$ 99.8+\%, KF 99.8+\%, RbF 99.8+\%, CsF 99.8+\%, N,N-dimethyl-formamide (DMF) $99.8+\%$, molecular sieve 4A 1/8 were purchased from J \& K Scientific Ltd. (China). Cis-1H,2H-octafluorocyclopentane (cis-F8A) $98.0+\%, 1 \mathrm{H}, 1 \mathrm{H}, 2 \mathrm{H}-$ 
heptafluorocyclopentane (F7A) 98.0+\%, 1,2,3-trichloropentafluorocyclopentene (F5-123) 98.0+\%, $\mathrm{CCl}_{3} \mathrm{~F}$ (CFC-11) 99.0+\% were purchased from Synquest Labs, Lnc. (USA). A mixture of 1,4-dichlorohexafluorocyclopentene (F6-14) and 1,3dichlorohexafluorocyclopentene (F6-13) (total purity: +98\%) was synthesized by the fluorine-chlorine exchange of F5-123 with KF in DMF [12], and 2.0\% Pd + $0.1 \% \mathrm{Bi} / \mathrm{PAF}$ (surface area $72.3 \mathrm{~m}^{2} / \mathrm{g}$ ) was used in the synthesis [13].

\subsection{Instrument}

The mass spectrometer was a GC-MS-QP2010 Ultra (Shimadzu). The column temperature program of GC-MS was as follows: $40^{\circ} \mathrm{C}$ for $4 \mathrm{~min} ; 15^{\circ} \mathrm{C} / \mathrm{min}$ to $230^{\circ} \mathrm{C}$; hold for $8 \mathrm{~min}$. Both the injection port and the thermal conductivity detector were maintained at $200^{\circ} \mathrm{C}$, and the carrier gas was $\mathrm{He}$ introduced at a rate of $10 \mathrm{ml} / \mathrm{min}$.

${ }^{19} \mathrm{~F}$ NMR spectra of the intermediates and products during the synthesis were recorded on a Bruker AVANCE 400 (400 MHz) NMR with CFC-11 as internal standards in $\mathrm{CDCl}_{3}$ at $25^{\circ} \mathrm{C}$. ${ }^{1} \mathrm{H}$ NMR spectra of the intermediates and products during the synthesis were recorded on a Bruker AVANCE $400(400 \mathrm{MHz})$ NMR in $\mathrm{CDCl}_{3}$ at $25^{\circ} \mathrm{C}$. A distillation tower with 3 meters long was used to rectify various products.

\subsection{Experiment Procedure}

Preparation of raw materials F7E- $1 \mathrm{H}$ and F7E-4H: $\left(\mathrm{NH}_{4}\right)_{2} \mathrm{CO}_{3}(0.50 \mathrm{~mol})$, tetra-n-butylammonium bromide $(0.05 \mathrm{~mol})$ and $500.0 \mathrm{ml}$ of $\mathrm{H}_{2} \mathrm{O}$ were placed into a $1000 \mathrm{~mL}$, three-necked, round-bottomed flask equipped with a thermometer and an agitating device. Then cis-F8A $(0.50 \mathrm{~mol})$ was added by drops into the above solution. Under magnetic stirring for $6 \mathrm{~h}$ at $50^{\circ} \mathrm{C}$, the organic phase of the products from the above system was dried with $4 \mathrm{~A}$ molecular sieve. Then, the organic phase was detected by ${ }^{19} \mathrm{~F}-\mathrm{NMR}$. The conversion of F8A was $89.3 \%$, and the yield of F7E-1H, F7E- $4 \mathrm{H}$ and F7E- $3 \mathrm{H}$ was $74.3 \%, 14.7 \%$ and $0.3 \%$, respectively. The above organic phase was directly rectified by a distillation tower with 3 meters long, and F6E-4H and F6E-1H were obtained. And F7E-4H was obtained by the following liquid-phase isomerization of F7E-3H promoted by fluoride anion.

Preparation of raw materials F6E-33H, F6E-14H and F6E-13H: A mixture of F6-14, F6-13 (molar ratio: $\mathrm{n}(\mathrm{F} 6-14) / \mathrm{n}(\mathrm{F} 6-13)=33 / 67)$, and $\mathrm{H}_{2}$ was supplied to the reactor made of Inconel through a vaporizer kept at $200^{\circ} \mathrm{C}$ under the conditions as follows: hydrogenation catalyst $2 \% \mathrm{Pd}+0.1 \% \mathrm{Bi} / \mathrm{AlF}_{3} 10 \mathrm{~mL}$, molar ratio $\mathrm{n}\left(\mathrm{H}_{2}\right) / \mathrm{n}(\mathrm{F} 6-13+\mathrm{F} 6-14)=2(\mathrm{~mol} / \mathrm{mol}), \mathrm{P}=0.1 \mathrm{MPa}$ and contact time $=13 \mathrm{~s}$. The products were washed by water, which separated the liquid-phase organic product from $\mathrm{HCl}$. Next, the liquid organic products were dried by a molecular sieve and then detected by ${ }^{19} \mathrm{~F}-\mathrm{NMR}$. The result was shown as follows: the total conversion of F6-13 and F6-14 was 87.3\%, and the yield of F6E-33H, F6E-13H, F6E-14H and F6E-44 was $16.8 \%, 45.1 \%, 10.6 \%$ and $2.5 \%$, respectively. The above 
organic phase was directly rectified by a distillation tower with 3 meters long, F6E-33H, F6E-14H and F6E-13H were obtained, respectively.

Preparation of raw materials F6E-12H and F6E-15H: $\left(\mathrm{NH}_{4}\right)_{2} \mathrm{CO}_{3}(0.50 \mathrm{~mol})$, tetra-n-butylammonium bromide $(0.05 \mathrm{~mol})$ and $500.0 \mathrm{ml}$ of $\mathrm{H}_{2} \mathrm{O}$ were placed into a $1000 \mathrm{~mL}$, three-necked, round-bottomed flask equipped with a thermometer and an agitating device. Then F7A $(0.50 \mathrm{~mol})$ was added by drops into the above solution. Under magnetic stirring for $6 \mathrm{~h}$ at $50^{\circ} \mathrm{C}$, the organic phase of the products from the above system was dried with $4 \mathrm{~A}$ molecular sieve. Then, the organic phase was detected by ${ }^{19} \mathrm{~F}-\mathrm{NMR}$. The conversion of F7A was $70.5 \%$, and the yield of $\mathrm{F} 6 \mathrm{E}-12 \mathrm{H}$, and $\mathrm{F} 6 \mathrm{E}-15 \mathrm{H}$ was $64.0 \%$, and $6.5 \%$, respectively. The above organic phase was directly rectified by a distillation tower with 3 meters long, F6E-12H and F6E-15H were obtained, respectively.

Liquid-phase isomerization of isomers of heptafluorocyclopentene: metal fluoride $(0.015 \mathrm{~mol})$ and $20.0 \mathrm{ml}$ of N.N-dimethylformamide were placed into a 50 $\mathrm{mL}$, three-necked, round-bottomed flask equipped with a thermometer, and an agitating device. Then F7E-3H, F7E-1H or F7E-4H (0.03 mol) was added by drops into the above solution. Under magnetic stirring for a certain time at room temperature $\left(25^{\circ} \mathrm{C}\right)$, the products from the above system were scrubbed with $100 \mathrm{~mL} \mathrm{H}_{2} \mathrm{O}$ to remove metal fluoride and N.N-dimethylformamide. Later, the products were dried with $4 \mathrm{~A}$ molecular sieve to obtain the organic phase of the product, which was detected by ${ }^{19} \mathrm{~F}-\mathrm{NMR}$. The results were shown in Table 1.

Table 1. Impact of catalyst on the isomerization of heptafluorocyclopentene $e^{a}$.

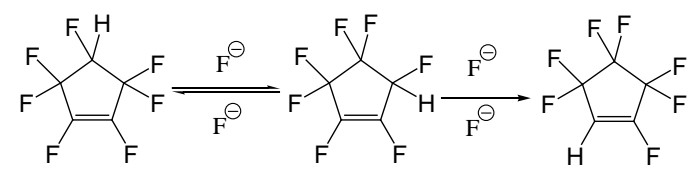

\begin{tabular}{|c|c|c|c|c|}
\hline \multirow[b]{2}{*}{ Material } & \multicolumn{2}{|r|}{ F7E-4H } & F7E-3H & \multirow[b]{2}{*}{ Amount of $\mathrm{F} 7 \mathrm{E}-3 \mathrm{H}^{\mathrm{b}} / \%$} \\
\hline & Catalyst & Amount of F7E- $4 \mathrm{H}^{\mathrm{b}} / \%$ & Amount of F7E-1H $1 \mathrm{H}^{\mathrm{b}} \%$ & \\
\hline F7E-3H & $\mathrm{LiF}$ & 2.1 & 0.7 & 97.2 \\
\hline F7E-3H & $\mathrm{NaF}$ & 4.0 & 0.8 & 95.2 \\
\hline F7E-3H & $\mathrm{KF}$ & 39.7 & 6.3 & 54.0 \\
\hline F7E-3H & $\mathrm{RbF}$ & 48.2 & 2.5 & 49.3 \\
\hline F7E-3H & $\mathrm{CsF}$ & 0.0 & 99.9 & 0.1 \\
\hline F7E-4H & $\mathrm{LiF}$ & 1.5 & 1.2 & 97.3 \\
\hline F7E-4H & $\mathrm{NaF}$ & 3.7 & 1.4 & 94.9 \\
\hline F7E-4H & $\mathrm{KF}$ & 37.8 & 8.9 & 53.3 \\
\hline F7E-4H & $\mathrm{RbF}$ & 49.0 & 4.3 & 46.7 \\
\hline F7E-4H & $\mathrm{CsF}$ & 11.5 & 84.8 & 3.7 \\
\hline F7E-1H & $\mathrm{LiF}$ & 0.1 & 94.7 & 5.2 \\
\hline F7E-1H & $\mathrm{NaF}$ & 0.1 & 99.8 & 0.1 \\
\hline
\end{tabular}

${ }^{a}$ Reaction conditions: F7E-1H, F7E-3H or F7E-4H: 0.03 mol, catalyst: 0.015 mol, Temperature $=25^{\circ} \mathrm{C}, \mathrm{DMF}$ $20 \mathrm{~mL}$, Time $=6 \mathrm{~h} .{ }^{\mathrm{b}}$ Amount was determined by ${ }^{19} \mathrm{~F}$ NMR versus a calibrated internal standard. 
Liquid-phase isomerization of isomers of hexafluorocyclopentene: metal fluoride $(0.015 \mathrm{~mol})$ and $20.0 \mathrm{ml}$ of N.N-dimethylformamide were placed into a 50 $\mathrm{mL}$, three-necked, round-bottomed flask equipped with a thermometer and an agitating device. Then F6E-33H, F6E-13H, F6E-14H, F6E-15H or F6E-12H (0.03 mol) was added by drops into the above solution. Under magnetic stirring for a certain time at room temperature $\left(25^{\circ} \mathrm{C}\right)$, the products from the above system were scrubbed with $100 \mathrm{~mL} \mathrm{H}_{2} \mathrm{O}$ to remove metal fluoride and N.N-dimethylformamide and were dried with $4 \mathrm{~A}$ molecular sieve to obtain the organic phase of the product. The organic phase of the product was detected by ${ }^{19} \mathrm{~F}-\mathrm{NMR}$. The results were shown in Table 2.

Table 2. Impact of catalyst on the isomerization of hexafluorocyclopentenea.

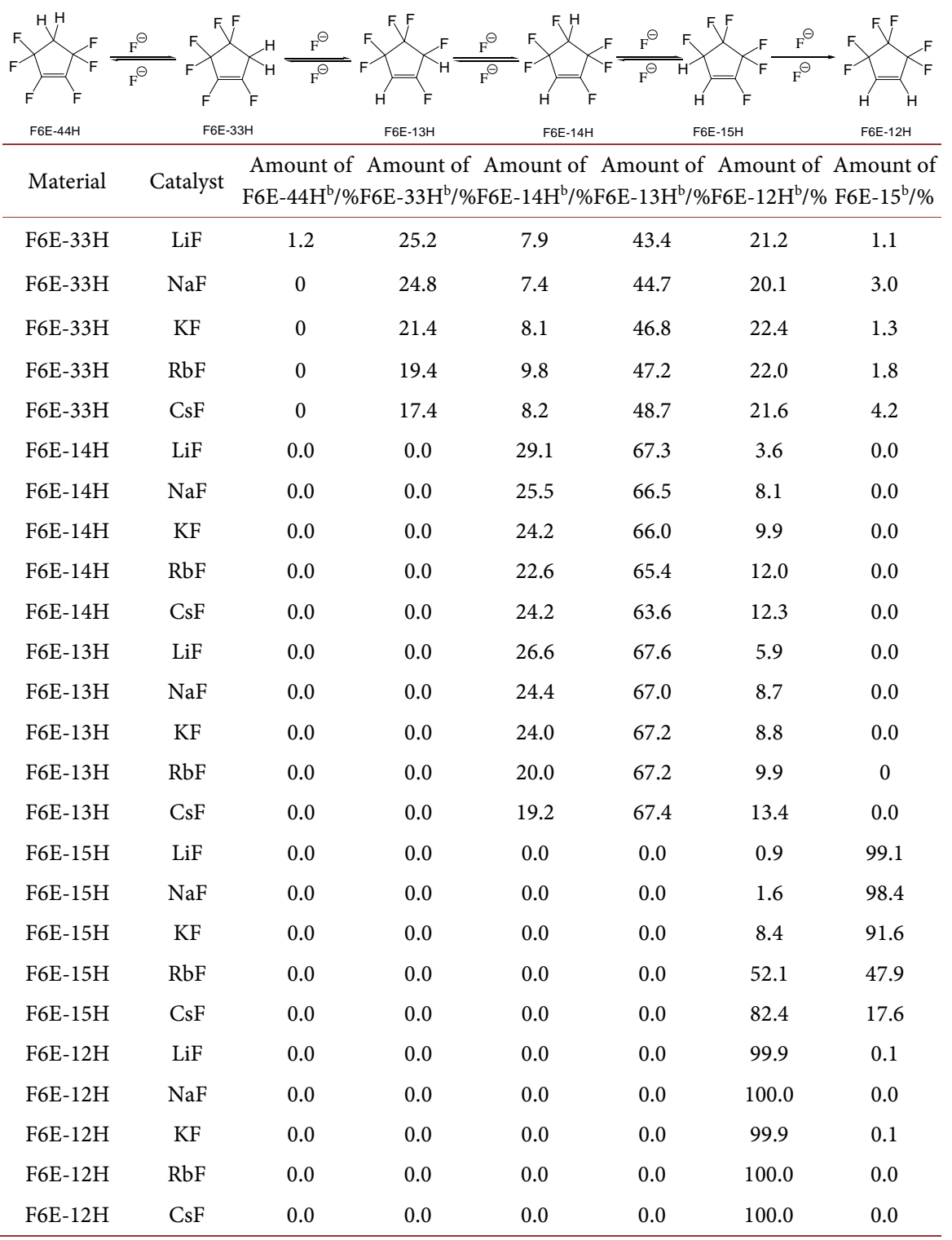

${ }^{a}$ Reaction conditions: F6E-33H, F6E-14H, F6E-13H, F6E-15H or F6E-12H: 0.03 mol, catalyst: 0.015 mol, Temperature $=25^{\circ} \mathrm{C}$, DMF $20 \mathrm{~mL}$, Time $=6 \mathrm{~h}$. ${ }^{\mathrm{b}}$ Amount was determined by ${ }^{19} \mathrm{~F}$ NMR versus a calibrated internal standard. 


\subsection{Analytic Results of and by-Products}

\subsubsection{F7E-1H}

Molecular structure:

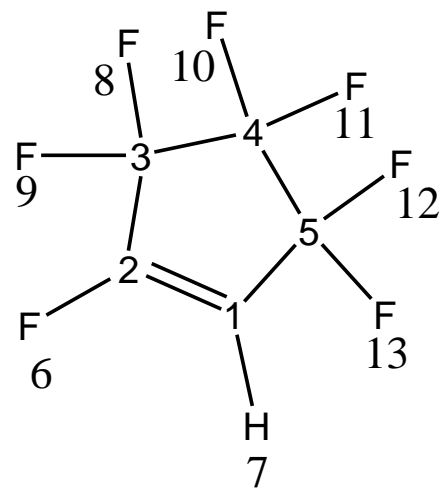

MS m/e: $194\left(\mathrm{M}^{+}\right) ; 175\left(\mathrm{M}^{+}-\mathrm{F}\right) ; 144\left(\mathrm{M}^{+}-\mathrm{CF}_{2}\right) ; 125\left(\mathrm{M}^{+}-\mathrm{CF}_{3}\right) ; 113\left(\mathrm{M}^{+}-\mathrm{C}_{2} \mathrm{~F}_{3}\right) ; 106$ $\left(\mathrm{M}^{+}-\mathrm{CF}_{4}\right) ; 87 \quad\left(\mathrm{M}^{+}-\mathrm{CF}_{5}\right) ; 75 \quad\left(\mathrm{M}^{+}-\mathrm{C}_{2} \mathrm{~F}_{5}\right) ; 69 \quad\left(\mathrm{M}^{+}-\mathrm{C}_{4} \mathrm{HF}_{4}\right) ; 56 \quad\left(\mathrm{M}^{+}-\mathrm{C}_{2} \mathrm{~F}_{6}\right) ; 44$ $\left(\mathrm{M}^{+}-\mathrm{C}_{3} \mathrm{~F}_{6}\right) ; 37\left(\mathrm{M}^{+}-\mathrm{C}_{2} \mathrm{~F}_{7}\right) ;{ }^{1} \mathrm{H}$ NMR $\left(400 \mathrm{MHz}, \mathrm{CDCl}_{3}\right) \delta 6.05(\mathrm{~s}, H 7,1 \mathrm{H}) ;{ }^{19} \mathrm{~F}$ NMR (377 MHz, Chloroform-d) $\delta-107.29$ (s, F12 and F13, 2F), -120.72 (s, F8 and $F 9,2 \mathrm{~F}),-124.83(\mathrm{~s}, F 7,1 \mathrm{~F}),-130.84$ (s, $F 10$ and $F 11,2 \mathrm{~F}$ ).

\subsubsection{F7E-3H}

Molecular structure:

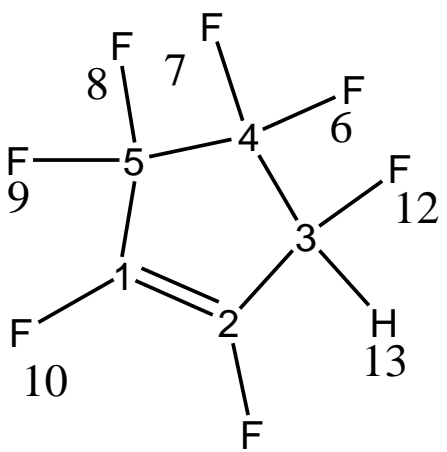

MS m/e: $194\left(\mathrm{M}^{+}\right) ; 175\left(\mathrm{M}^{+}-\mathrm{F}\right) ; 144\left(\mathrm{M}^{+}-\mathrm{CF}_{2}\right) ; 125\left(\mathrm{M}^{+}-\mathrm{CF}_{3}\right) ; 113\left(\mathrm{M}^{+}-\mathrm{C}_{2} \mathrm{~F}_{3}\right)$; $106\left(\mathrm{M}^{+}-\mathrm{CF}_{4}\right) ; 93\left(\mathrm{M}^{+}-\mathrm{C}_{2} \mathrm{HF}_{4}\right) ; 87\left(\mathrm{M}^{+}-\mathrm{CF}_{5}\right) ; 75\left(\mathrm{M}^{+}-\mathrm{C}_{2} \mathrm{~F}_{5}\right) ; 69\left(\mathrm{M}^{+}-\mathrm{C}_{4} \mathrm{HF}_{4}\right) ; 56$ $\left(\mathrm{M}^{+}-\mathrm{C}_{2} \mathrm{~F}_{6}\right) ; 51\left(\mathrm{M}^{+}-\mathrm{C}_{4} \mathrm{~F}_{5}\right) ; 37\left(\mathrm{M}^{+}-\mathrm{C}_{2} \mathrm{~F}_{7}\right) ;{ }^{1} \mathrm{H}$ NMR $\left(400 \mathrm{MHz}, \mathrm{CDCl}_{3}\right) \delta 5.43(\mathrm{~d}, \mathrm{~J}$ $=28.4 \mathrm{~Hz}, H 13,1 \mathrm{H}) ;{ }^{19} \mathrm{~F}$ NMR $(377 \mathrm{MHz}$, Chloroform-d) $\delta-110.26(\mathrm{dm}, \mathrm{J}=$ $127.80, F 6,1 \mathrm{~F}),-118.81(\mathrm{dm}, \mathrm{J}=126.48 \mathrm{~Hz}, F 7,1 \mathrm{~F}),-122.06(\mathrm{dm}, \mathrm{J}=124.60$ $\mathrm{Hz}, F 8,1 \mathrm{~F}),-128.38(\mathrm{dm}, \mathrm{J}=127.61 \mathrm{~Hz}, F 9,1 \mathrm{~F}),-137.72(\mathrm{~m}, F 10,1 \mathrm{~F}),-194.240$ $(\mathrm{m}, F 10,1 \mathrm{~F})$.

\subsubsection{F7E-4H}

Molecular structure: 


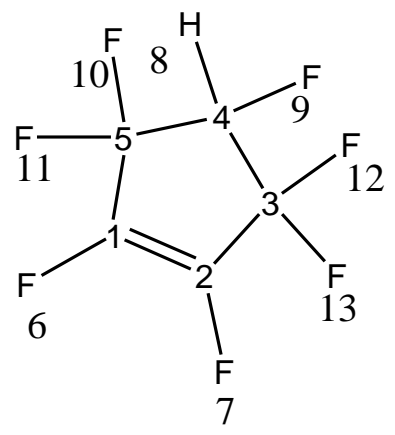

MS m/e: $194\left(\mathrm{M}^{+}\right) ; 175\left(\mathrm{M}^{+}-\mathrm{F}\right) ; 144\left(\mathrm{M}^{+}-\mathrm{CF}_{2}\right) ; 125\left(\mathrm{M}^{+}-\mathrm{CF}_{3}\right) ; 113\left(\mathrm{M}^{+}-\mathrm{C}_{2} \mathrm{~F}_{3}\right)$; $106\left(\mathrm{M}^{+}-\mathrm{CF}_{4}\right) ; 93\left(\mathrm{M}^{+}-\mathrm{C}_{2} \mathrm{HF}_{4}\right) ; 87\left(\mathrm{M}^{+}-\mathrm{CF}_{5}\right) ; 75\left(\mathrm{M}^{+}-\mathrm{C}_{2} \mathrm{~F}_{5}\right) ; 69\left(\mathrm{M}^{+}-\mathrm{C}_{4} \mathrm{HF}_{4}\right) ; 56$ $\left(\mathrm{M}^{+}-\mathrm{C}_{2} \mathrm{~F}_{6}\right) ; 51\left(\mathrm{M}^{+}-\mathrm{C}_{4} \mathrm{~F}_{5}\right) ; 37\left(\mathrm{M}^{+}-\mathrm{C}_{2} \mathrm{~F}_{7}\right) ;{ }^{1} \mathrm{H}$ NMR $\left(400 \mathrm{MHz}, \mathrm{CDCl}_{3}\right) \delta 5.05(\mathrm{~d}, \mathrm{~J}$ $=24.8 \mathrm{~Hz}, H 8,1 \mathrm{H}) ;{ }^{19} \mathrm{~F}$ NMR $(377 \mathrm{MHz}$, Chloroform-d) $\delta-109.98(\mathrm{dm}, \mathrm{J}=$ $124.79, F 11$ and $F 13,2 \mathrm{~F}),-115.09$ (dm, J = 128.75, F10 and $F 12,2 \mathrm{~F}),-152.27$ $(\mathrm{m}, F 6$ and $F 7,2 \mathrm{~F}),-214.47(\mathrm{dm}, \mathrm{J}=24.32, F 7,1 \mathrm{~F})$.

\subsubsection{F6E-12H}

Molecular structure:

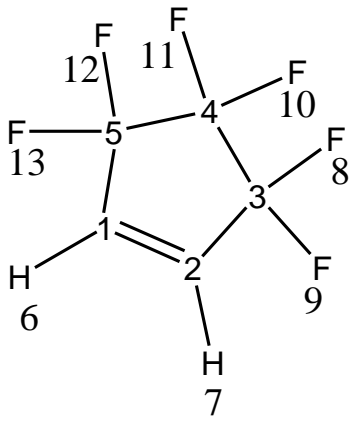

MS m/e: $176\left(\mathrm{M}^{+}\right) ; 157\left(\mathrm{M}^{+}-\mathrm{F}\right) ; 137\left(\mathrm{M}^{+}-\mathrm{HF}_{2}\right) ; 126\left(\mathrm{M}^{+}-\mathrm{CF}_{2}\right) ; 113\left(\mathrm{M}^{+}-\mathrm{CHF}_{2}\right)$; $107\left(\mathrm{M}^{+}-\mathrm{CF}_{3}\right) ; 100\left(\mathrm{M}^{+}-\mathrm{C}_{2} \mathrm{H}_{2} \mathrm{~F}_{2}\right) ; 88\left(\mathrm{M}^{+}-\mathrm{CF}_{4}\right) ; 75\left(\mathrm{M}^{+}-\mathrm{C}_{2} \mathrm{HF}_{4}\right) ; 69\left(\mathrm{M}^{+}-\mathrm{CF}_{5}\right) ; 57$ $\left(\mathrm{M}^{+}-\mathrm{C}_{2} \mathrm{~F}_{5}\right) ; 51\left(\mathrm{M}^{+}-\mathrm{C}_{4} \mathrm{HF}_{4}\right) ; 38\left(\mathrm{M}^{+}-\mathrm{C}_{2} \mathrm{~F}_{6}\right) ;{ }^{1} \mathrm{H}$ NMR $\left(400 \mathrm{MHz}, \mathrm{CDCl}_{3}\right) \delta 6.56(\mathrm{~m}$, $H 6$ and $H 7,2 \mathrm{H}) ;{ }^{19} \mathrm{~F}$ NMR $(377 \mathrm{MHz}$, Chloroform-d) $\delta-109.92$ (m, F8, $\boldsymbol{F}$, $F 12$ and $F 13,4 \mathrm{~F}),-132.46$ (m, F10 and $F 11,2 \mathrm{~F})$.

\subsubsection{F6E-13H}

Molecular structure:

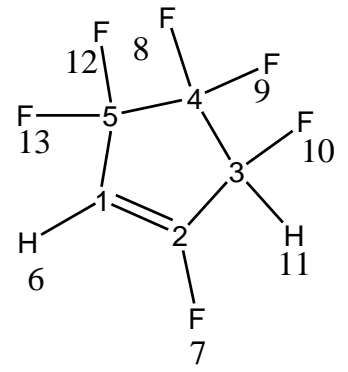


MS m/e: $176\left(\mathrm{M}^{+}\right) ; 157\left(\mathrm{M}^{+}-\mathrm{F}\right) ; 137\left(\mathrm{M}^{+}-\mathrm{HF}_{2}\right) ; 126\left(\mathrm{M}^{+}-\mathrm{CF}_{2}\right) ; 113\left(\mathrm{M}^{+}-\mathrm{C}_{2} \mathrm{~F}_{2} \mathrm{H}\right)$; $107\left(\mathrm{M}^{+}-\mathrm{CF}_{3}\right) ; 94\left(\mathrm{M}^{+}-\mathrm{C}_{2} \mathrm{HF}_{3}\right) ; 88\left(\mathrm{M}^{+}-\mathrm{CF}_{4}\right) ; 75\left(\mathrm{M}^{+}-\mathrm{C}_{2} \mathrm{HF}_{4}\right) ; 69\left(\mathrm{M}^{+}-\mathrm{CF}_{5}\right) ; 57$ $\left(\mathrm{M}^{+}-\mathrm{C}_{2} \mathrm{~F}_{5}\right) ; 51\left(\mathrm{M}^{+}-\mathrm{C}_{4} \mathrm{HF}_{4}\right) ; 44\left(\mathrm{M}^{+}-\mathrm{C}_{3} \mathrm{HF}_{5}\right) ; 38\left(\mathrm{M}^{+}-\mathrm{C}_{2} \mathrm{~F}_{6}\right) ;{ }^{1} \mathrm{H}$ NMR $(400 \mathrm{MHz}$, $\left.\mathrm{CDCl}_{3}\right) \delta 5.81(\mathrm{~s}, H 6,1 \mathrm{H}), 5.31$ (ddd, J = $26.60 \mathrm{~Hz}, 8.80 \mathrm{~Hz}, 5.20 \mathrm{~Hz}, H 11,1 \mathrm{H}$ ); ${ }^{19}$ F NMR (377 MHz, Chloroform-d) $\delta-100.99(\mathrm{dm}, \mathrm{J}=127.80 \mathrm{~Hz}, F 12,1 \mathrm{~F})$, $-110.68(\mathrm{dd}, \mathrm{J}=134.86 \mathrm{~Hz}, 7.16 \mathrm{~Hz}, F 13,1 \mathrm{~F}),-111.78(\mathrm{~m}, F 7,1 \mathrm{~F}),-120.81(\mathrm{dm}$, $\mathrm{J}=125.35 \mathrm{~Hz}, F 8,1 \mathrm{~F}),-128.55(\mathrm{dm}, \mathrm{J}=125.16 \mathrm{~Hz}, \boldsymbol{F}, 1 \mathrm{~F}),-196.84(\mathrm{dd}, \mathrm{J}=$ $53.16 \mathrm{~Hz}, 26.01 \mathrm{~Hz}, F 10,1 \mathrm{~F})$.

\subsubsection{F6E-14H}

Molecular structure:

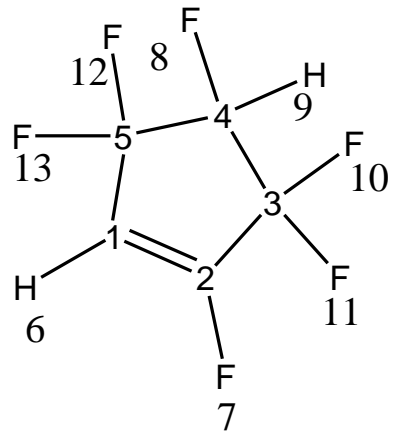

MS m/e: $176\left(\mathrm{M}^{+}\right) ; 157\left(\mathrm{M}^{+}-\mathrm{F}\right) ; 137\left(\mathrm{M}^{+}-\mathrm{HF}_{2}\right) ; 126\left(\mathrm{M}^{+}-\mathrm{CF}_{2}\right) ; 107\left(\mathrm{M}^{+}-\mathrm{CF}_{3}\right) ; 93$ $\left(\mathrm{M}^{+}-\mathrm{C}_{2} \mathrm{H}_{2} \mathrm{~F}_{3}\right) ; 88\left(\mathrm{M}^{+}-\mathrm{CF}_{4}\right) ; 75\left(\mathrm{M}^{+}-\mathrm{C}_{2} \mathrm{HF}_{4}\right) ; 69\left(\mathrm{M}^{+}-\mathrm{CF}_{5}\right) ; 57\left(\mathrm{M}^{+}-\mathrm{C}_{2} \mathrm{~F}_{5}\right) ; 51$ $\left(\mathrm{M}^{+}-\mathrm{C}_{4} \mathrm{HF}_{4}\right) ; 44\left(\mathrm{M}^{+}-\mathrm{C}_{3} \mathrm{HF}_{5}\right) ; 37\left(\mathrm{M}^{+}-\mathrm{C}_{2} \mathrm{HF}_{6}\right) ;{ }^{1} \mathrm{H} \mathrm{NMR}\left(400 \mathrm{MHz}, \mathrm{CDCl}_{3}\right) \delta 5.91$ (s, $H 6,1 \mathrm{H}$ ), 5.02 (dt-quartet, J = $24.00 \mathrm{~Hz}, 10.00 \mathrm{~Hz}, 2.00 \mathrm{~Hz}, H \boldsymbol{H}, 1 \mathrm{H}$ ); ${ }^{19} \mathrm{~F}$ NMR (377 MHz, Chloroform-d) $\delta-94.81(\mathrm{dm}, \mathrm{J}=262.02 \mathrm{~Hz}, F 12,1 \mathrm{~F}),-104.73$ (dd, J $=261.64 \mathrm{~Hz}, 11.31 \mathrm{~Hz}, F 13,1 \mathrm{~F}),-110.95(\mathrm{ddm}, \mathrm{J}=268.05 \mathrm{~Hz}, 4.52 \mathrm{~Hz}, F 10,1 \mathrm{~F})$, $-118.11(\mathrm{dd}, \mathrm{J}=255.61 \mathrm{~Hz}, 15.08 \mathrm{~Hz}, F 11,1 \mathrm{~F}),-151.16(\mathrm{~s}, F 7,1 \mathrm{~F}),-210.05(\mathrm{dm}$, $\mathrm{J}=49.76 \mathrm{~Hz}, F 8,1 \mathrm{~F})$.

\subsubsection{F6E-15H}

Molecular structure:

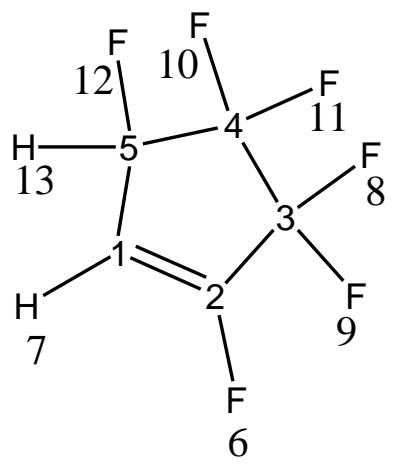

MS m/e: $176\left(\mathrm{M}^{+}\right) ; 157\left(\mathrm{M}^{+}-\mathrm{F}\right) ; 137\left(\mathrm{M}^{+}-\mathrm{HF}_{2}\right) ; 126\left(\mathrm{M}^{+}-\mathrm{CF}_{2}\right) ; 113\left(\mathrm{M}^{+}-\mathrm{CHF}_{2}\right)$; 
$107\left(\mathrm{M}^{+}-\mathrm{CF}_{3}\right) ; 93\left(\mathrm{M}^{+}-\mathrm{CH}_{2} \mathrm{~F}_{3}\right) ; 88\left(\mathrm{M}^{+}-\mathrm{CF}_{4}\right) ; 75\left(\mathrm{M}^{+}-\mathrm{C}_{2} \mathrm{HF}_{4}\right) ; 69\left(\mathrm{M}^{+}-\mathrm{CF}_{5}\right) ; 57$ $\left(\mathrm{M}^{+}-\mathrm{C}_{2} \mathrm{~F}_{5}\right) ; 51\left(\mathrm{M}^{+}-\mathrm{C}_{4} \mathrm{HF}_{4}\right) ; 38\left(\mathrm{M}^{+}-\mathrm{C}_{2} \mathrm{~F}_{6}\right) ;{ }^{1} \mathrm{H} \mathrm{NMR}\left(400 \mathrm{MHz}, \mathrm{CDCl}_{3}\right) \delta 6.00(\mathrm{~m}$, $H 7,2 \mathrm{H}) ; 5.45$ (ddd, $\mathrm{J}=9.60 \mathrm{~Hz}, 5.60 \mathrm{~Hz}, 1.60 \mathrm{~Hz}, H 13,2 \mathrm{H}) ;{ }^{19} \mathrm{~F}$ NMR $(377$ $\mathrm{MHz}$, Chloroform-d) $\delta-113.48(\mathrm{dm}, \mathrm{J}=130.01 \mathrm{~Hz}, F 8,1 \mathrm{~F}),-120.40(\mathrm{dm}, \mathrm{J}=$ $67.48 \mathrm{~Hz}, F 9,1 \mathrm{~F}),-125.70(\mathrm{dm}, \mathrm{J}=68.41 \mathrm{~Hz}, F 10,1 \mathrm{~F}),-129.11(\mathrm{~m}, F 6,1 \mathrm{~F})$, $-186.14(\mathrm{dm}, \mathrm{J}=56.93 \mathrm{~Hz} F 12,1 \mathrm{~F})$.

\subsubsection{F6E-33H}

Molecular structure:<smiles></smiles>

MS m/e: $176\left(\mathrm{M}^{+}\right) ; 157\left(\mathrm{M}^{+}-\mathrm{F}\right) ; 137\left(\mathrm{M}^{+}-\mathrm{HF}_{2}\right) ; 126\left(\mathrm{M}^{+}-\mathrm{CF}_{2}\right) ; 113\left(\mathrm{M}^{+}-\mathrm{C}_{2} \mathrm{~F}_{2} \mathrm{H}\right)$; $107\left(\mathrm{M}^{+}-\mathrm{CF}_{3}\right) ; 94\left(\mathrm{M}^{+}-\mathrm{C}_{2} \mathrm{HF}_{3}\right) ; 88\left(\mathrm{M}^{+}-\mathrm{CF}_{4}\right) ; 75\left(\mathrm{M}^{+}-\mathrm{C}_{2} \mathrm{HF}_{4}\right) ; 69\left(\mathrm{M}^{+}-\mathrm{CF}_{5}\right) ; 57$ $\left(\mathrm{M}^{+}-\mathrm{C}_{2} \mathrm{~F}_{5}\right) ; 51\left(\mathrm{M}^{+}-\mathrm{C}_{4} \mathrm{HF}_{4}\right) ; 44\left(\mathrm{M}^{+}-\mathrm{C}_{3} \mathrm{HF}_{5}\right) ; 38\left(\mathrm{M}^{+}-\mathrm{C}_{2} \mathrm{~F}_{6}\right) ;{ }^{1} \mathrm{H}$ NMR $(400 \mathrm{MHz}$, $\left.\mathrm{CDCl}_{3}\right) \delta 3.08$ (m, H12 and H13, 2H); ${ }^{19} \mathrm{~F} \mathrm{NMR} \mathrm{(377} \mathrm{MHz,} \mathrm{Chloroform-d)} \delta$ $113.65(\mathrm{tdd}, \mathrm{J}=10.93 \mathrm{~Hz}, 5.66 \mathrm{~Hz}, 1.89 \mathrm{~Hz}, F 12$ and $F 13,2 \mathrm{~F}),-117.21(\mathrm{tt}, \mathrm{J}=$ $12.44 \mathrm{~Hz}, 3.77 \mathrm{~Hz}, F 8$ and $F 9,2 \mathrm{~F}),-128.16(\mathrm{~m}, F 7,1 \mathrm{~F}),-163.36(\mathrm{~m}, F 6,1 \mathrm{~F})$.

\subsubsection{F6E-44H}

Molecular structure:

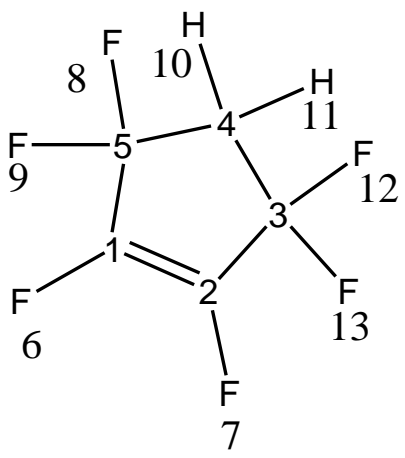

MS m/e: $176\left(\mathrm{M}^{+}\right) ; 157\left(\mathrm{M}^{+}-\mathrm{F}\right) ; 137\left(\mathrm{M}^{+}-\mathrm{HF}_{2}\right) ; 126\left(\mathrm{M}^{+}-\mathrm{CF}_{2}\right) ; 113\left(\mathrm{M}^{+}-\mathrm{C}_{2} \mathrm{~F}_{2} \mathrm{H}\right)$; $107\left(\mathrm{M}^{+}-\mathrm{CF}_{3}\right) ; 94\left(\mathrm{M}^{+}-\mathrm{C}_{2} \mathrm{HF}_{3}\right) ; 88\left(\mathrm{M}^{+}-\mathrm{CF}_{4}\right) ; 75\left(\mathrm{M}^{+}-\mathrm{C}_{2} \mathrm{HF}_{4}\right) ; 69\left(\mathrm{M}^{+}-\mathrm{CF}_{5}\right) ; 57$ $\left(\mathrm{M}^{+}-\mathrm{C}_{2} \mathrm{~F}_{5}\right) ; 51\left(\mathrm{M}^{+}-\mathrm{C}_{4} \mathrm{HF}_{4}\right) ; 44\left(\mathrm{M}^{+}-\mathrm{C}_{3} \mathrm{HF}_{5}\right) ; 38\left(\mathrm{M}^{+}-\mathrm{C}_{2} \mathrm{~F}_{6}\right) ;{ }^{1} \mathrm{H}$ NMR $(400 \mathrm{MHz}$, $\mathrm{CDCl}_{3}$ ) $\delta 2.94$ (quintet-t, $\mathrm{J}=9.60 \mathrm{~Hz}, 1.60 \mathrm{~Hz}, H 10$ and $\left.H 11,2 \mathrm{H}\right) ;{ }^{19} \mathrm{~F} \mathrm{NMR}(377$ $\mathrm{MHz}$, Chloroform-d) $\delta-62.18(\mathrm{~m}, F 8, F 9, F 12$ and $F 13,4 \mathrm{~F}),-136.39(\mathrm{~m}, F 6$ 
and $F 7,2 \mathrm{~F}$ ).

Note: The number ( $\boldsymbol{n})$ of $\boldsymbol{F} \boldsymbol{n}$ or $\boldsymbol{H} \boldsymbol{n}$ in NMR data is in agreement with the number in the molecular structure.

\section{Results and Discussion}

In $\mathrm{DMF}$, promoted by various alkali metal fluoride such as $\mathrm{LiF}, \mathrm{NaF}, \mathrm{KF}, \mathrm{RbF}$ or $\mathrm{CsF}$ at $25^{\circ} \mathrm{C}$ for 6 hours, F7E-3H was isomerized into F7E-1H and F7E-4H (See Table 1). The conversion of F7E-3H increased with the increasing atomic number of alkali metal. The larger the atomic number of alkali metal, the stronger the reactivity of alkali metal fluoride in halogen exchange [14]. And the efficacy of the alkali fluorides with respect to replacement reactions ranked in the sequence: $\mathrm{CsF}>\mathrm{RbF}>\mathrm{KF}>\mathrm{NaF}>\mathrm{LiF}$ [15] [16] [17]. Therefore, CsF owned the highest activity in the isomerization of F7E-3H. In addition, the solubility of the alkali metal fluoride in DMF affect the reactivity at a certain, but not only reason. In DMF, RbF (1.05 mM) has a higher solubility than CsF (0.60 mM) in DMF [18], but its reactivity is weaker than $\mathrm{CsF}$ in the isomerization of F7E-3H. The isomerizations of c5-HFOs belong to a kind of special halogen exchange (fluorine-fluorine exchange), which are probably not simply solution reactions but probably occurring on the surface. The system usually involve an excess of the solid metal fluoride present and, indeed, in some reactions it has been observed that the amount of solid metal fluoride is important [14]. Therefore, alkali metal fluoride was always excess in the isomerizations of c5-HFOs.

As shown in Table 1, F7E-4H was isomerized into F7E-1H and F7E-3H, while F7E-1H was isomerized into F7E-3H and F7E-4H in a small amount. This indicated that there existed difficultiesin the isomerization of F7E-1H into F7E-3H and F7E-4H probably due to the high energy barrier between F7E-1H and $\mathrm{F} 7 \mathrm{E}-3 \mathrm{H}$ (or F7E-4H). Thus, F7E-1H was the absolutely preferred isomer.

As listed in Table 2, isomers of hexafluorocyclopentene can be interconverted in DMF in the presence of alkali metal fluoride, including $3 \mathrm{H}, 3 \mathrm{H}$-hexafluorocyclopentene $(\mathrm{F} 6 \mathrm{E}-33 \mathrm{H}), 4 \mathrm{H}, 4 \mathrm{H}$-hexafluorocyclopentene $(\mathrm{F} 6 \mathrm{E}-44 \mathrm{H}), 3 \mathrm{H}, 3 \mathrm{H}-$ hexafluorocyclopentene (F6E-33H), 1H,5H-hexafluoro-cyclopentene (F6E-15H), $1 \mathrm{H}, 4 \mathrm{H}$-hexafluorocyclopentene $(\mathrm{F} 6 \mathrm{E}-14 \mathrm{H}), 1 \mathrm{H}, 3 \mathrm{H}$-hexafluorocyclopentene (F6E$13 \mathrm{H})$ and $1 \mathrm{H}, 2 \mathrm{H}$-hexafluorocyclopentene $(\mathrm{F} 6 \mathrm{E}-12 \mathrm{H})$. F6E-33H was isomerized into $\mathrm{F} 6 \mathrm{E}-44 \mathrm{H}, \mathrm{F} 6 \mathrm{E}-15 \mathrm{H}, \mathrm{F} 6 \mathrm{E}-14 \mathrm{H}, \mathrm{F} 6 \mathrm{E}-13 \mathrm{H}$ and $\mathrm{F} 6 \mathrm{E}-12 \mathrm{H}$, and the conversion of $\mathrm{F} 6 \mathrm{E}-33 \mathrm{H}$ was $82.6 \%$, the yield of $\mathrm{F} 6 \mathrm{E}-13 \mathrm{H}$ was $48.7 \%$ in the presence of $\mathrm{CsF}$. F6E-14H was converted to F6E-13H and F6E-12H, while F6E-13H was turned into $\mathrm{F} 6 \mathrm{E}-14 \mathrm{H}$ and $\mathrm{F} 6 \mathrm{E}-12 \mathrm{H}$. In the isomerization of $\mathrm{F} 6 \mathrm{E}-14 \mathrm{H}$ or $\mathrm{F} 6 \mathrm{E}-13 \mathrm{H}$, neither $\mathrm{F} 6 \mathrm{E}-44 \mathrm{H}$ nor $\mathrm{F} 6 \mathrm{E}-33 \mathrm{H}$ was produced. It will be explained in the discussion part of this paper. F6E-15H was isomerized into $\mathrm{F} 6 \mathrm{E}-12 \mathrm{H}$, and the conversion of $\mathrm{F} 6 \mathrm{E}-15 \mathrm{H}$ was $82.4 \%$, the yield of $\mathrm{F} 6 \mathrm{E}-12 \mathrm{H}$ was $82.4 \%$ when promoted by CsF. F6E-12H was difficult to be isomerized into F6E-15H. Thus, F6E- $12 \mathrm{H}$ is the absolutely preferred isomer among the various isomers of hexafluorocyclopentene. In addition, the selectivity of various c5-HFOs can be 
controlled when the different alkali metal fluoride is chosen as a catalyst.

Based on the results of our experiments, the possible reaction path of liquidphase isomerizations of c5-HFOs was proposed as follows (Scheme 1):

1) Alkali metal fluoride was partially soluble in DMF [18]. This provided the naked fluoride anions to catalyze the isomerizations of c5-HFOs.

2) In $\mathrm{DMF}$, the $\mathrm{C}=\mathrm{C}$ of $\mathrm{F} 7 \mathrm{E}-4 \mathrm{H}$ underwent nucleophilic attack by the incoming fluoride anion to give a tetrahedral intermediate I-34, which proceeded to the product F7E-3H by elimination of fluoride anion. The whole process belonged to an allylic syn-1-addition/3-elimination of fluoride anion [12] [19] [20], which was reversible. This is because the syn-elimination reaction of the five membered ring is easier to form a co-planar transition state, which reduces the energy barrier and makes the reaction easy to occur [21].

3) In DMF, the $\mathrm{C}=\mathrm{C}$ of $\mathrm{F} 7 \mathrm{E}-3 \mathrm{H}$ underwent nucleophilic attack by the incoming fluoride anion to give a tetrahedral intermediate I-13, which proceeded to the product F7E-1H by elimination of fluoride anion. The above

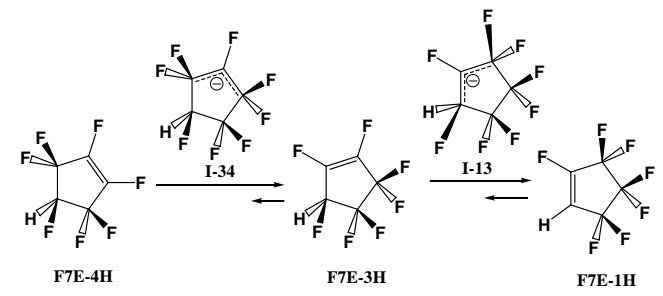

(a)

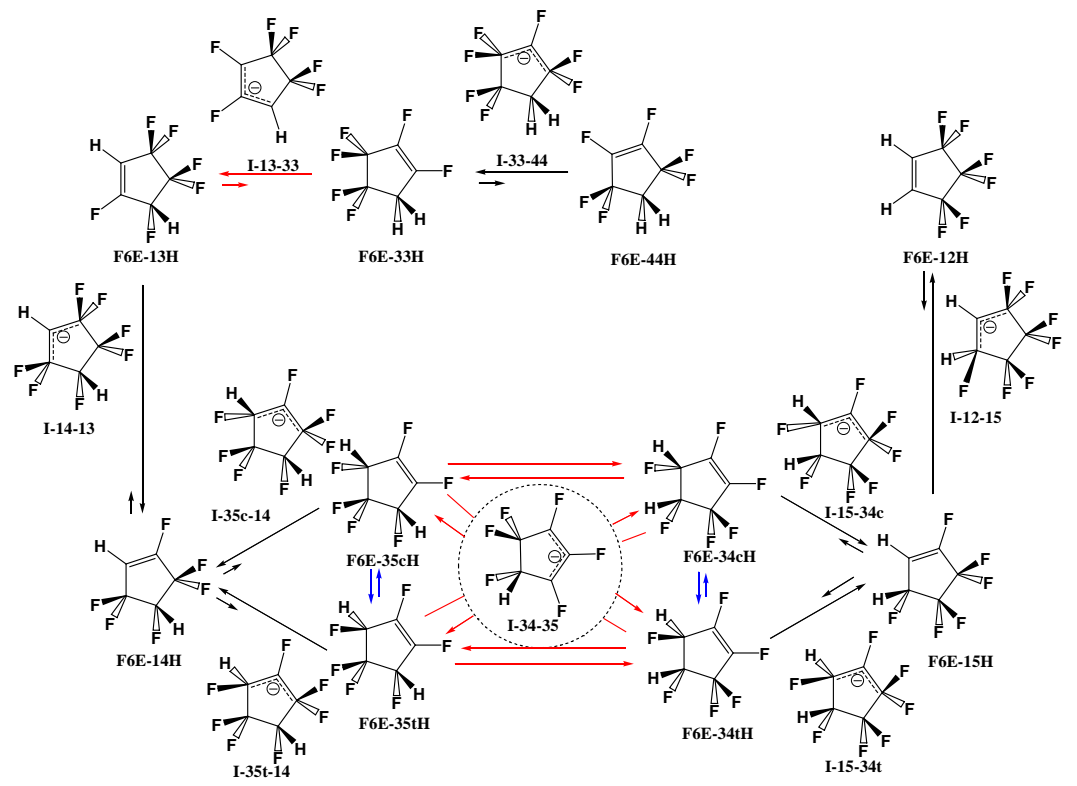

(b)

Scheme 1. The possible reaction path of Isomerization reactions of c5-HFOs promoted by fluoride anion (Black arrows: allylic syn-addition/elimination of fluoride anion; Red arrows: fluoride anion-assisted deprotonation/protonation; Blue arrows: geometric isomerization). (a) Isomerization of hydrofluorocyclopentene; (b) Isomerization of hexafdfluorocyclopentene. 
process belonged to an allylic syn-1-addition/3-elimination of fluoride anion [12] [19] [20], which was not a chemical equilibrium.

4) In DMF, the $\mathrm{C}=\mathrm{C}$ of $\mathrm{F} 6 \mathrm{E}-44 \mathrm{H}$ underwent nucleophilic attack by the incoming fluoride anion to give a tetrahedral intermediate I-33-44, which proceeded to the product $\mathrm{F} 6 \mathrm{E}-33 \mathrm{H}$ by elimination of fluoride anion. It belonged to an allylic syn-1-addition/3-elimination of fluoride anion [12] [19] [20], which was a chemical equilibrium.

5) In DMF, F6E-33H underwent deprotonation with fluoride anion in 3position on the $\mathrm{C}\left(\mathrm{sp}^{3}\right)$ atom to give an intermediate I-13-33 with the release of $\mathrm{HF}$, which proceeded to the product F6E-13H by protonation of I-13-33 with HF in 1-position on the carbon atom with the release of fluoride anion [22]. This was a chemical equilibrium. Here, fluoride anion acted as a catalyst.

6) In DMF, the $\mathrm{C}=\mathrm{C}$ of $\mathrm{F} 6 \mathrm{E}-13 \mathrm{H}$ underwent nucleophilic attack by the incoming fluoride anion to give a tetrahedral intermediate I-14-13, which proceeded to the product F6E-14H by elimination of fluoride anion. The process belonged to a syn-1-addtion/3-elimination of fluoride anion [12] [19] [20], which was a chemical equilibrium.

7) In DMF, the $\mathrm{C}=\mathrm{C}$ of $\mathrm{F} 6 \mathrm{E}-14 \mathrm{H}$ underwent nucleophilic attack by the incoming fluoride anion to give tetrahedral intermediates I-35c-14 (cis-isomer) and I-35t-14 (trans-isomer), which proceeded to the products $\mathrm{F} 6 \mathrm{E}-35 \mathrm{cH}$ and F6E-35tH by elimination of fluoride anion. The whole process belonged to an allylic syn-1-addition/3-elimination of fluoride anion [12] [19] [20], which was a chemical equilibrium; Then, $\mathrm{F} 6 \mathrm{E}-35 \mathrm{cH}$ or $\mathrm{F} 6 \mathrm{E}-35 \mathrm{tH}$ underwent deprotonation with fluoride anion in 3-position on the $\mathrm{C}\left(\mathrm{sp}^{3}\right)$ atom to give an intermediate I-34-35 with the release of HF, which proceeded to the product $\mathrm{F} 6 \mathrm{E}-34 \mathrm{cH}$ or F6E-34tH by protonation of I-34-35 with $\mathrm{HF}$ in 1-position on the carbon atom with the release of fluoride anion [21], and the $\mathrm{C}=\mathrm{C}$ of $\mathrm{F} 6 \mathrm{E}-34 \mathrm{cH}$ or $\mathrm{F} 6 \mathrm{E}-34 \mathrm{tH}$ underwent nucleophilic attack by the incoming fluoride anion to give tetrahedral intermediates I-15-34c (cis-isomer) and I-15-34t (trans-isomer), which proceeded to the products $\mathrm{F} 6 \mathrm{E}-15 \mathrm{H}$ by elimination of fluoride anion. The whole process belonged to an allylic syn-1-addition/3-elimination of fluoride anion [12] [19] [20], which was not a chemical equilibrium.

8) In DMF, the $\mathrm{C}=\mathrm{C}$ of $\mathrm{F} 6 \mathrm{E}-15 \mathrm{H}$ underwent nucleophilic attack by the incoming fluoride anion to give a tetrahedral intermediate I-12-15, which proceeded to the product F6E-12H by elimination of fluoride anion. The whole process belonged to a syn-1-addtion/3-elimination of fluoride anion [12] [19] [20], which was not a chemical equilibrium.

In the above mechanisms of the isomerizations of c5-HFOs, the fluoride anion-assisted deprotonation/protonation is one important process. Its occurrence is closely related to the position of the hydrogen atom on the fiveelement ring (See Scheme 2(a)). When hydrogen atom is in 3-position, 4-position or 5-position on $\mathrm{C}\left(\mathrm{sp}^{3}\right)$ atom in $\mathrm{c5}$-HFOs, c5-HFOs easily react with fluoride anion via deprotonation/protonation, and the hydrogen atom can 


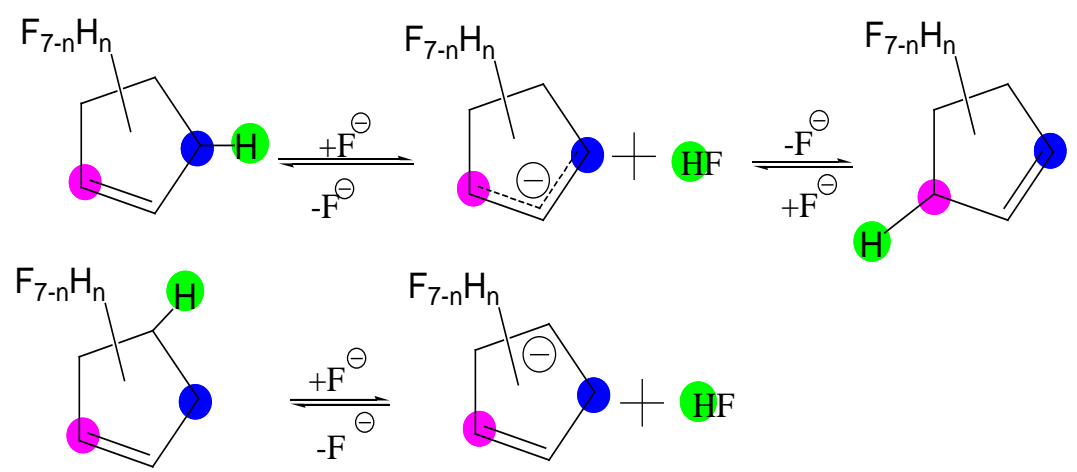

(a)
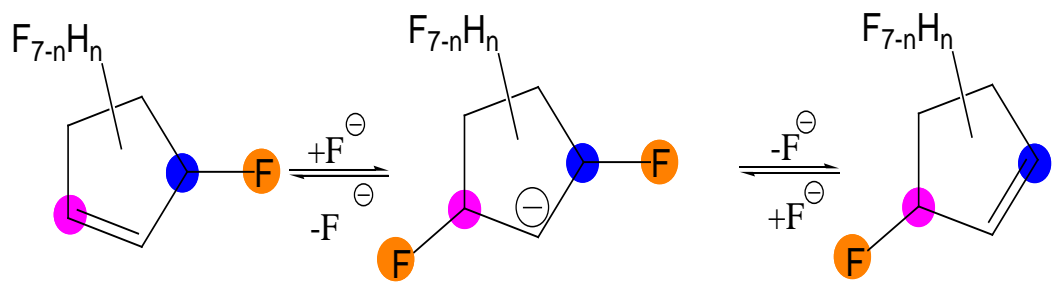

(b)

Scheme 2. Two processes of isomerization of c5-HFOs promoted by fluoride anion. (a) Deprotonation/protonation $(0=0$ or 1$)$; (b) Addition/elimination $(n=1$ or 2$)$.

transfer on the five-membered ring due to the rearrangement of the pentacyclic anions, but the hydrogen atom in 4-position maintains its position because that the pentacyclic anions cannot rearrange. When hydrogen atom is only in 1-position or/and 2-position on $\mathrm{C}\left(\mathrm{sp}^{2}\right)$ atom in c5-HFOs, c5-HFOs difficultly react with fluoride anion via deprotonation/protonation due to the strong attraction of $\mathrm{C}=\mathrm{C}$ double bond towards fluorine atom. The allylic syn-addition/ elimination of fluoride anion is another important process in the isomerizations of c5-HFOs (See Scheme 2(b)). The fluorine atom can transfer on the fivemembered ring via the allylic syn-addition/elimination of fluoride anion due to the rearrangement of the pentacyclic anions.

Now, we explain why neither F6E-44H nor F6E-33H was found in the isomerization of $\mathrm{F} 6 \mathrm{E}-14 \mathrm{H}$ or $\mathrm{F} 6 \mathrm{E}-13 \mathrm{H}$. In DMF, the c5-HFO that contained $\mathrm{C}\left(\mathrm{sp}^{3}\right)-\mathrm{H}$, such as $\mathrm{F} 6 \mathrm{E}-13 \mathrm{H}, \mathrm{F} 6 \mathrm{E}-14 \mathrm{H}$ orF6E-35cH, underwent deprotonation with fluoride anion in 3 (4, or 5)-position on the $\mathrm{C}\left(\mathrm{sp}^{3}\right)$ atom to give an intermediate with the release of HF (See Scheme 2(a)). According to the Gibbs free energies of the formation $\mathrm{HF}_{2}^{-}$anion and the ionization of $\mathrm{HF}_{2}^{-}$from the quantum chemical calculations using Gaussian09 (See Table 3), once HF captured excess fluoride anion, with the formation of a very stable anion $\mathrm{HF}_{2}{ }^{-}$, the protonation of the intermediate was terminated because the anion $\mathrm{HF}_{2}{ }^{-}$was incapable of neither catalyzing isomerizations nor returning the proton back. Thus, the reaction was "frozen". When quenched with $\mathrm{H}_{2} \mathrm{O}$ in the experiments, the intermediate returned to its raw material. Therefore, neither F6E-33H nor F6E-44H was found in the isomerization of F6E-14H or F6E-13H. 
Table 3. Gibbs free energies calculations for the formation of $\mathrm{HF}_{2}^{-}$and the ionization of $\mathrm{HF}_{2}^{-}$using Gaussian 09 (in DMF, $25^{\circ} \mathrm{C}$ ).

\begin{tabular}{cc}
\hline Reaction & Gibbs free energy $\left(\mathrm{kcal} \cdot \mathrm{mol}^{-1}\right)$ \\
\hline 1) $\mathrm{HF}+\mathrm{F}^{-}=\mathrm{HF}_{2}^{-}$ & -28.1783 \\
2) $\mathrm{HF}=\mathrm{H}^{+}+\mathrm{F}^{-}$ & +190.5848 \\
3) $\mathrm{HF}_{2}^{-}=\mathrm{H}^{+}+2 \mathrm{~F}^{-}$ & +218.7631 \\
\hline
\end{tabular}

\section{Conclusion}

In the isomerization of c5-HFOs promoted by fluoride anion, two main processes are responsible for the interconversion of the isomers. An allylic syn-addition/ elimination of fluoride anion that does not change the mutual positions of hydrogen atoms but is responsible for transfers of fluorine atoms, and a fluoride anion-assisted deprotonation/protonation which does not change the mutual positions of fluorine atoms but is responsible for transfers of hydrogen atoms. It was also revealed that $\mathrm{HF}_{2}^{-}$anion in the deprotonation can inhibit the protonation. This is probably the reason that neither F6E-33h nor F6E-44H was found in the isomerization of F6E-13H or F6E-14H.

\section{Acknowledgements}

This research is supported by Ministry of Environmental Protection of the People's Republic of China.

\section{References}

[1] Sugimoto, T. (2015) High-Purity 1h-Heptafluorocyclopentene. European Patent No. 2960224A1.

[2] Elsheikh, M.Y., Bonnet, P. and Chen, B.B. (2010) Process for the Manufacture of Hydrofluoroolefins. US Patent No. 20100185030.

[3] Sugimoto, T. (2011) Method of Isomerizing Heptafluorocyclopentene, and Use Thereof. Japan Patent No. 2011105625.

[4] Feast, W.J., Perry, D.R.A. and Stephens, R. (1966) Fluorocyclopentanes-V Lithium Aluminium Hydride Reduction of Octafluoro-1,2-Dichlorohexafluoro-, and 1Chloroheptafluoro-Cyclopentene and 1H,2-Chloro-Octafluorocyclohexene. Tetrahedron, 22, 433-439. https://doi.org/10.1016/0040-4020(66)80007-8

[5] Nose, M., Komatsu, Y., Sugiyama, A. and Shibanuma, T. (2011) Process for Preparing 2,3,3,3-Tetrafluoropropene and 1,3,3,3-Tetrafluoropropene. US Patent No. 20110137090 .

[6] Tung, H.S. and Wang, H.Y. (2011) Method for Making Hexafluoro-2-Butene. World Patent No. 2011119370.

[7] Cottrell, S.A., Tung, H.S. and Wang, H. (2010) Manufacturing Process for HFO1234ze. US Patent No. 20100022809.

[8] Okamoto, S., Takada, N., Sakyu, F., Kitamoto, T., Tonomura, T. and Kanai, M. (2015) Method for Producing cis-1,3,3,3-Tetrafluoropropene. US Patent No. 20150112103.

[9] Nappa, M.J. (2010) Catalytic Isomerization between E and Z Isomers of 1,2,3,3,3 
Pentafluoropropene Using Aluminum Catalyst. US Patent No. 20100197980.

[10] Nappa, M.J. (2013) Catalytic Isomerization of 2,3,3,3-Tetrafluoropropene. US Patent No. 20130253234.

[11] Wang, H., Pokrovski, K.A., Tung, H.S. and Puy, M.V.D. (2010) Isomerization of 1,1,3,3-Tetrafluoropropene. US Patent No. 20100185027.

[12] Zhang, C.P., Qing, F.Y., Quan, H.D. and Sekiya, A. (2016) Synthesis of 1,1,2,2,3,3,4Heptafluorocyclopentane as a New Generation of Green Solvent. Journal of Fluorine Chemistry, 181, 11-16. https://doi.org/10.1016/j.jfluchem.2015.10.012

[13] Jia, X.Q., Zhou, X.M., Quan, H.D., Tamura, M. and Sekiya, A. (2011) Preparation of cis-1,1,1,4,4,4-Hexafluorobut-2-Ene by Cis-Selective Semi-Hydrogenation of Perfluoro-2-Butyne. Journal of Fluorine Chemistry, 132, 1188-1193. https://doi.org/10.1016/j.jfluchem.2011.06.004

[14] Chambers, R.D. (1973) Fluorine in Organic Chemistry. John Wiley \& Sons, Inc., New York, London, Sydney, Toronto, 21-23.

[15] Miloš, H. (1972) Chemistry of Organic Fluorine Compounds. 2nd Edition, Halsted Press, John Wiley \& Sons, New York, London, Sydney, Toronto, 112.

[16] Moilliet, J.S. (1994) Organofluorine Chemistry. Springer, New York, 195-219. https://doi.org/10.1007/978-1-4899-1202-2_9

[17] Watcher (1994) Kirk-Othmer Encyclopedia of Chemical Technology. 4th Edition, Wiley, New York, 11, 267.

[18] Wynn, D.A., Roth, M.M. and Pollard, B.D. (1984) The Solubility of Alkali-Metal Fluorides in Non-Aqueous Solvents with and without Crown Ethers, as Determined by Flame Emission Spectrometry. Talanta, 31, 1036-1040. https://doi.org/10.1016/0039-9140(84)80244-1

[19] Faber, K., Fessner, W.D. and Turner, N. (2015) Biocatalysis in Organic Synthesis. Georg Thieme Verlag KG Stuttgart, New York, 265.

[20] Zhang, C.P., Hu, R.Z., Qing, F.Y. and Quan, H.D. (2016) Isomerization of Halogenated Cyclopentene over a $\mathrm{NH}_{4} \mathrm{~F}$ Catalyst. ChemCatChem, 8, 1474-1478.

https://doi.org/10.1002/cctc.201600055

[21] DePuy, C.H., Thurn, R.D. and Morris, G.F. 1962 Concerted Bimolecular Eliminations and Some Comments on the Effect of Dihedral Angle on E2 Reactions. Journal of the American Chemical Society, 84, 1314-1315. https://doi.org/10.1021/ja00866a058

[22] Muhammad, S., Xu, H., Janjua, M.R.S.A., Su, Z. and Nadeem, M. (2010) Quantum Chemical Study of Benzimidazole Derivatives to Tune the Second-Order Nonlinear Optical Molecular Switching by Proton Abstraction. Physical Chemistry Chemical Physics, 12, 4791-4799. https://doi.org/10.1039/b924241d 